See discussions, stats, and author profiles for this publication at: https://www.researchgate.net/publication/233216864

\title{
Cannabis use and psychotic experiences in an international sample of undergraduate students
}

Article in (Psychosis) Psychological, Social and Integrative Approaches · June 2011

DOI: 10.1080/17522439.2010.495413

CITATIONS

READS

0

48

3 authors, including:

Thomas Richardson

Solent NHS Trust

110 PUBLICATIONS 1,674 CITATIONS

SEE PROFILE

Some of the authors of this publication are also working on these related projects:

Project Finances in Bipolar View project

IMAGEN View project 


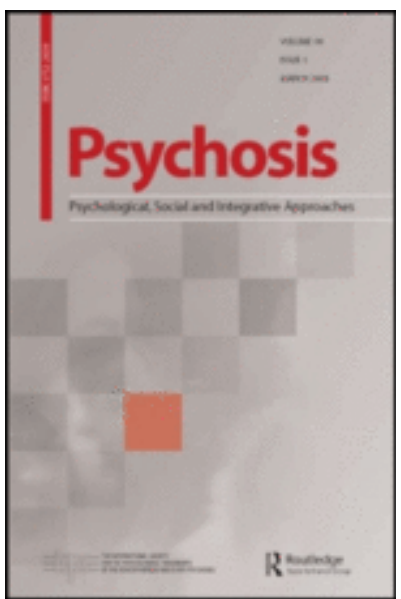

\section{Cannabis use and psychotic symptoms in an international sample of undergraduate students.}

\begin{tabular}{|r|l|}
\hline Journal: & Psychosis \\
\hline Manuscript ID: & Draft \\
\hline Manuscript Type: & Research Article \\
\hline Keywords: & Cannabis, Delusions, Drug abuse, Epidemiology, Hallucinations \\
\hline \multicolumn{2}{|}{} \\
\hline
\end{tabular}

\section{s scholaroNE" \\ Manuscript Central}




\title{
Cannabis use and psychotic symptoms in an international sample of undergraduate students.
}

\author{
Thomas Richardson ${ }^{\mathrm{a}, \mathrm{b}} *$, Alanna Gallagher ${ }^{\mathrm{c}} \& \operatorname{Hugh}_{\text {Garavan }}^{\mathrm{b}}$ \\ ${ }^{a}$ Mental Health Research and Development Unit, University of Bath, U.K. \\ b School of Psychology and Institute of Neuroscience, Trinity College Dublin, \\ Ireland. \\ ${ }^{\mathrm{c}}$ Parents Plus Charity Trust, Dublin, Ireland. \\ * = Corresponding author. Thomas Richardson, Room 2.15, M.H.R.D.U., School for \\ Health, University of Bath, Bath, B.A.N.E.S., BA2 7AY, U.K. Telephone: +44 \\ (0)1225 385428. Email: T.H.Richardson@bath.ac.uk.
}

Word count $=4,916$

Funding sources- No funding obtained.

Declaration of conflict of interests- None. 


\begin{abstract}
Background: Cannabis use may increase psychotic symptoms in the general population. However few studies have examined this relationship in undergraduate students despite high levels of cannabis use in this population. Aims: To examine relationships been self-reported cannabis use and psychotic symptoms in an international sample of undergraduate students.

Method: 334 undergraduate students (Mean age $=22.2$ years; $85.2 \%$ female, $14.8 \%$ male) from Britain, Ireland, Canada, The United States, Australia and New Zealand took part in the study. Participants completed an author constructed questionnaire measuring cannabis use, and questions on psychosis from the Diagnostic Interview Schedule (DIS-P) as adapted for self-report format.

Results: Overall, 53.1\% ( $\mathrm{n}=178)$ of participants had used cannabis at some point in their lives and $20.1 \%(n=67)$ were currently using cannabis. Current cannabis use and frequency of current use were associated with elevated levels of psychotic symptoms. Age of first use was also significantly related to psychotic symptoms, but in the reverse direction expected, with those who began using cannabis later in life having higher levels of psychosis. Lifetime usage was not significantly related to psychotic symptoms.
\end{abstract}

Conclusion: Cannabis use appears to be associated with higher levels of psychotic symptoms in undergraduate students.

Keywords: Cannabis, marijuana, psychosis, psychotic, students. 


\section{Introduction}

Cannabis is the most commonly used illicit drug in the world, with an estimated 162 million current users worldwide (United Nations, Office on Drugs and Crime, 2006). In recent years evidence is emerging that such use is associated with a number of adverse effects on health, including relationships with a number of mental health problems such as psychosis (Hall \& Degenhardt, 2009).

A number of studies have documented a relationship between cannabis use and psychotic symptoms and schizophrenia. Co-morbidity is high between the two, with half of all first-episode psychosis patients having a history of cannabis use (Barnett et al., 2007). Cannabis use in such patients can lead to increased hallucinatory behaviour and grandiosity (Katz et al., 2010), and elevated risk of relapse (Hides et al., 2006). Cannabis also may reduce the age of onset of psychotic symptoms, though causality is difficult to establish (Compton \& Ramsay, 2009).

There is evidence to suggest that cannabis increases the risk of later psychosis in the general population. A review by Arsenault et al (2004) concluded that cannabis doubles the risk of later schizophrenia. Van Os et al (2002) conducted a 3 year longitudinal study of more than 4,000 individuals from the general population of the Netherlands, finding that cannabis use predicted later psychotic symptoms, and more than half of all psychosis was related to cannabis use. Additional research has found a similar relationship even after controlling for potential confounding variables such as predisposition to psychosis, socioeconomic status, gender, and other drug use (Henquet et al., 2005; Hides et al., 2009; Miettunen et al., 2008).

Studies have shown a dose-dependent association between cannabis use and subclinical psychotic symptoms, with increasingly heavy cannabis use leading to increasingly severe psychosis (Ferdinand et al., 2005; Hides et al., 2006). Frequency of use (Compton \& Ramsay, 2009), and strength of cannabis (DiForti et al., 2009), are also related to subsequent risk of psychosis. Starting to use cannabis at a younger age has been shown to increase the risk of later psychotic symptoms (Arsenault et al., 2002; Stefanis et al., 2005). 
A number of studies have documented high levels of cannabis use in university students (Arria et al., 2008; Caldeira et al., 2008; Hope, Dring \& Dring, 2005). In the USA, Arria et al (2008) found that cannabis was used by nearly $60 \%$ of students by their final year, and that some continued use from before college whilst others started use at university. Similarly, in Ireland, a survey found that $37 \%$ of students had used cannabis in the past 12 months, and 20\% in the past 30 days (Hope, Dring \& Dring, 2005). This was considerably higher than use in non students of the same age (National Advisory Committee on Drugs, 2003).

Despite this, there has been little research examining the relationship between cannabis use and psychotic symptoms in this population. Verdoux et al (2003) found that in students cannabis intoxication induced acute psychotic-like symptoms such as unusual perceptions. Similarly, Barkus \& Lewis (2008) found that in university students those with higher levels of schizotypy were more likely to experience acute psychotic symptoms during and after cannabis use. However, there has been little research examining the relationship between cannabis use and chronic psychotic symptoms in students outside the immediate effects of acute intoxication. The aim of this study was therefore to study relationships between self reported cannabis use history and psychotic symptoms in an international sample of undergraduate students. It was hypothesised that cannabis users would display increased levels of psychotic symptoms such as hallucinations and delusions, and that an increased frequency of use, a higher level of consumption, and lower age of initial use would all be related to increased levels of psychosis.

\section{Methods}

\section{Design and Procedure}

This study was granted ethical approval by Trinity College's School of Psychology Research Ethics committee. A cross-sectional design of a non-clinical sample was used. An international undergraduate sample was obtained by emails being sent to university psychology department staff in the U.K., Ireland, Canada, Australia, New Zealand, and the U.S. This email explained the purposes of the research to staff, who then had the option to forward this email on to their students. A link was placed in the email to a website, where the measures were completed and consent and debriefing 
forms were provided. Taking part in this was voluntary and no compensation was offered. Participation was completely anonymous and subjects could leave the survey at any time. Due to the recruitment method used, the response rate obtained is unknown.

\section{Measures}

To measure psychotic symptoms, the questions on psychosis from the Diagnostic Interview Schedule (DIS-P) were used, as adapted by Caspi et al (2005) to a selfreport format. These questions measure a participant's history of psychotic symptoms with 24 questions requiring either 'Yes' or 'No' responses to questions such as "Do you ever/have you ever...hear things or voices that other people cannot hear". There are two subscales for this measure: 'Hallucinatory experiences' and 'Delusional beliefs'. Total scores range from 0 to 24 , with an increasingly high score representing increasingly severe psychotic symptoms.

Participants completed a self-report drug use history questionnaire, which included detailed questions about the participant's cannabis use. Specifically, participants were asked whether they had ever used cannabis and whether they still used cannabis. Age of first use, frequency of use, perceived addiction and estimated consumption were also examined.

\section{Participants}

The cohort examined here consists of undergraduate students who completed all questions of the DIS-P, and whose nationality was Irish, British, Canadian, U.S., New Zealander or Australian. This left a total of 335 participants. The sample was $85.2 \%$ $(n=282)$ female and $14.8 \%(n=49)$ male. The age of the sample ranged from 18 to 65 with a mean age of 22.2 years old $(\mathrm{SD}=5.73)$. The nationalities of the sample were 37\% ( $\mathrm{n}=124)$ British, 27.2\% ( $\mathrm{n}=91)$ Irish, 15.8\% ( $\mathrm{n}=53)$ U.S., 9.3\% ( $\mathrm{n}=31)$ New Zealander, 7.2\% (n=24) Australian and 3.6\% (n=12) Canadian.

\section{Statistical analysis}

A number of variables from the drug use questionnaire were categorical, e.g. "Have you ever used cannabis?" with a response "Yes" or "No". However, there were also a number of continuous variables such as age of first use of cannabis. These were 
predominantly not normally distributed, and were therefore transformed into categorical variables. For example, age of first use of cannabis was transformed into 'under the age of 17 ' or 'aged 17 and above'. Therefore, the independent variables were all categorical. The dependent variables were the total scores and two subscales for the DIS-P. These were all non-normally distributed, thus non-parametric MannWhitney independent samples t-tests were used. As it was hypothesised that those using drugs would show increased psychotic symptoms, one-tailed tests were used.

\section{Results}

Total scores on the DIS-P ranged from 0 to 22 out of 24 , with a median of 1 . Overall, $41.8 \%(n=140)$ of the sample scored 0 on the DIS-P (see Figure 1). Scores on the 'Hallucinatory Experiences' subscale ranged from 0 to 9 out of 9, with a median of 0 . Scores on the 'Delusional Beliefs' subscale ranged from 0 to 14 out of 15 , with a median of 0 . Table I summarises the cannabis use variables examined in this study. Table I also shows these variables broken down by gender, with significantly higher lifetime and past year consumption in male participants.

\section{**Insert Figure I and Table I here**}

\section{Cannabis use and psychotic symptoms}

A series of Mann-Whitney non-parametric independent samples t-tests were conducted to test for relationships between cannabis use and psychotic symptoms. Those currently using cannabis $(n=67)$ had a significantly higher score on the 'Hallucinatory experiences' subscale than those not currently using cannabis $(n=266)$; $\mathrm{Z}=-1.923, \mathrm{p}<.05$, one-tailed. Amongst those currently using cannabis, there was no difference between those who had been heavy users (10 or more 'joints') of cannabis in the past year $(n=34)$ compared to those who had been low users (less than 10 'joints') of cannabis in the past year $(n=28)$ on the DIS-P total; $Z=-.141, p>.05$, onetailed. Those who had used cannabis at some point in their lives $(n=178)$ did not differ from those who had never used cannabis $(n=157)$ on the DIS=P total; $Z=-.041, p>.05$. 
In participants who had used cannabis at some points in their lives, those who described their frequency of cannabis use as 'weekly' $(n=16)$ scored significantly higher than those who descried their use as 'yearly' $(n=71)$ on the DIS-P total; $Z=-$ 1.730, $\mathrm{p}<.05$, one-tailed, and 'Hallucinatory experiences' subscale; $\mathrm{Z}=-2.539, \mathrm{p}<.01$, one-tailed. Those who described their frequency of cannabis use as 'weekly' $(n=16)$ scored significantly higher than those who descried their use as 'monthly' $(n=33)$ on the DIS-P total; $\mathrm{Z}=-2.106, \mathrm{p}<.05$, one-tailed, and 'Hallucinatory experiences' subscale; $\mathrm{Z}=-2.892, \mathrm{p}<.01$, one-tailed.

Those who began using cannabis at age 17 or older $(n=74)$ had a significantly higher score than those who began using cannabis at younger than age $17(n=102)$ on the DIS-P total; $\mathrm{Z}=-2.012, \mathrm{p}<.05$, one-tailed, 'Hallucinatory experiences' subscale; $\mathrm{Z}=-$ 1.727, $\mathrm{p}<.05$, two-tailed, and 'Delusional beliefs' subscale; $\mathrm{Z}=-1.802, \mathrm{p}<.05$, onetailed. Additional analyses were completed to try to determine whether age of first use was confounded with any other cannabis use variables. The proportion of participants currently using cannabis was similar for those who began using cannabis younger than age $17(38.6 \%, n=39)$ compared to those who began using cannabis at age 17 or older $(37.8 \%, \mathrm{n}=28)$. A Mann-Whitney non-parametric independent samples t-test also revealed that those who began using cannabis before they were 17 years old had significantly higher lifetime consumption than those who began using cannabis at age 17 or older; $\mathrm{Z}=-2.423, \mathrm{p}<.01$, one-tailed.

\section{Discussion}

This study aimed to examine the relationship between cannabis use and psychotic symptoms in an international sample of undergraduate students. Self-reported use revealed high levels of cannabis use in this population, with more than half using cannabis at some point in their lives, and more than a fifth currently using the drug. Whilst this is high it is perhaps lower than previous estimates of prevalence of cannabis use in students (Arria et al., 2008; Caldeira et al., 2008). The age of first use varied considerably, with an average of age 16. This is similar to that seen in other studies; for example, Caldeira et al. (2008) found an average of cannabis use initiation of 15.7 years in a student sample. It may be that age of first use varies considerably in this sample due to exposure opportunities presented at university; Arria et al (2008) 
found that the majority of students were offered cannabis whilst at university and that many who had never used cannabis previously began doing so at university. In this study, some of those who used the drug did so frequently and were heavy users. This is in line with previous research which documents higher levels of problematic cannabis use and cannabis use disorders in students compared to the general population of adults of the same age (Caldeira et al., 2008). However, the majority appeared to use cannabis relatively infrequently and with relatively low doses, and very few people considered themselves to be addicted. This appears to represent a recreational pattern of use, which has previously been identified as common in students (Hammersley \& Leon, 2006). These results may however under-estimate the true levels of cannabis use in students as this sample was predominantly female, and research has shown that cannabis use is more common in male students (Hope et al., 2005). Additionally, in the current study, male students used significantly more cannabis than their female peers.

Overall the majority of students in this sample showed no or very few symptoms of psychosis. However, cannabis use appeared to increase the risk of experiencing such symptoms, with a number of cannabis use variables being related to scores representing overall psychotic symptoms, and hallucinations and delusions specifically. Those currently using cannabis had higher levels of hallucinatory symptoms than those who were not using cannabis, in line with other findings (Hides et al., 2009). This was not, however, the case for delusions or general psychotic symptoms. Hallucinatory type experiences such as perceptual alterations have been previously shown to be elevated in those currently using cannabis (Hides et al., 2009). However they have also been demonstrated whilst under the influence of cannabis (D'Souza et al., 2004; Stirling et al., 2008; Verdoux et al., 2003), thus, it may be that in this sample these may be acute hallucinatory symptoms experienced whilst intoxicated, rather than chronic psychotic symptoms. This is a problem in much research in the area, as many studies do not take into consideration the short-term effects of cannabis intoxication (McLaren et al., 2010). However it has been suggested that experiencing psychotic symptoms whilst under the influence may predict future chronic psychosis (Stirling et al., 2008). Increased frequency of use was related to increased levels of general psychotic symptoms and hallucinatory symptoms, in line with previous research (Compton et al., 2009; Van Os et al., 2002). 
This suggests that students who move from occasional, perhaps recreational, cannabis use to more frequent chronic use are at a greater risk of experiencing psychotic symptoms. It should however be noted that for the majority of students in this sample, the psychotic symptoms reported here are probably sub-clinical. Nonetheless, such sub-clinical psychosis increases the risk of future clinical psychotic episodes (Van Os et al., 2009).

Amongst those currently using cannabis, extent of use in the past year was not related to psychotic symptoms, at odds with previous research (Ferdinand et al., 2005; Hides et al., 2006). Thus it appears that whether or not students were currently using cannabis was an important predictor of psychotic symptoms, but level of consumption was not related to such symptoms. However, as previously mentioned increased frequency of use was related to increased psychotic symptoms in this study, suggesting that dose-response effects occur when measured by frequency of use rather than level of consumption. Although few participants in this study considered themselves to be addicted, those who used more frequently may represent individuals who are problematic cannabis users. Such problematic use has previously been found to predict increased psychotic symptoms (Fergusson et al., 2003).

In this sample there was also a statistically significant relationship between age of first use of cannabis and psychotic symptoms. However the observed relationship was not in the direction hypothesised, with those who began using cannabis at an older age having more psychotic symptoms than those who began using cannabis when they were younger. Previous research has found that early use increases the risk of psychosis (Arsenault et al., 2002; Barnett et al., 2007; Stefanis et al., 2004). Exactly why the reverse was found in this data set is unclear. It may be that those who began using earlier use less cannabis now compared to those with later onset, however in this study there was no difference in current use based on age of first use, and those who began using earlier had in fact used more cannabis over their lifetime. Thus those who begin using earlier are as likely to still be using, and have used more over the lifetime, yet they still they have lower levels of psychotic symptoms. It may be that those who use cannabis at an earlier age are less prone to psychosis initially; a recent review of 10 studies suggests that vulnerability to psychosis influences the relationships between cannabis use and psychosis (McLaren et al., 2010). However it 
is not possible to explore this possibility in this sample. Different reasons for use may account for this; those who begin using cannabis later may be using cannabis for different reasons, for example they may be more likely to be self-medicating for emerging psychotic symptoms. Evidence suggests that different reasons for using cannabis in students are related to differing levels of mental health problems (Beck et al., 2009). Finally, as previously discussed participants may be reporting the acute affects of cannabis intoxication rather than more chronic psychosis. It may be that those who begin using cannabis at an earlier age have developed a greater tolerance to the psychoactive effects of cannabis and are less likely to experience psychotic like symptoms with use. This has previously been suggested as an explanation for research demonstrating higher levels of psychotic symptoms in those who use cannabis less frequently (Richardson \& Gallagher, 2010).

As discussed above, those currently using cannabis displayed increased levels of psychosis. However, those who had used cannabis at some point in their lives did not differ in terms of psychotic symptoms compared to those who had never used cannabis. This goes against other findings, for example Hides et al (2009) found that any lifetime use was associated with increased psychotic symptoms. However other research suggests that current use, rather than lifetime use, is related to psychosis (DiForti et al., 2009). This suggests that current cannabis use predicts psychotic symptoms, whereas lifetime cannabis use is not related to psychosis. Thus psychotic symptoms may be due to recent cannabis use rather than the cumulative effects of long-term exposure. This again raises the possibility that the symptoms described here are acute and may represent the psychotropic effects of cannabis intoxication. However it may be that those still using cannabis are more chronic, long-term users of the drug who are therefore more likely to experience adverse effects.

In the sample a number of cannabis use variables were related to increased levels of psychosis in students. However causality in this instance is hard to establish. It may be that cannabis use increased psychotic symptoms in this sample; there is evidence from longitudinal studies that cannabis use at baseline predicts psychotic symptoms at follow-up (Henquet et al., 2005; Van Os et al., 2002). However this current study was not longitudinal, so it is not possible to determine whether cannabis predicts future psychotic symptoms. Alternatively, it may be that observed relationships occur 
because those who have higher levels of psychotic symptoms are more likely to use cannabis, perhaps to self-medicate; Schofield et al (2006) found that many patients with psychosis used cannabis to self-medicate the negative symptoms of the illness. In this study it is not clear when the self-reported psychotic symptoms occurred; therefore it is not possible to tell if these symptoms were present before cannabis use was initiated. It is also possible that both of these possibilities are correct; Ferdinand et al (2005) found that cannabis use predicted later psychotic symptoms, but psychotic symptoms also predicted future cannabis use. They suggest that the relationship may therefore be bi-directional; cannabis may induce psychotic symptoms as well as be used for self-medication of psychosis (Ferdinand et al., 2005). The issue of whether cannabis is a causal risk factor is a complicated and controversial, and whilst whilst there is evidence for a causal association, it is unclear whether cannabis can induce psychotic illnesses by itself (McLaren et al., 2010).

A number of additional limitations need to be considered. First, the sample used here is relatively small when compared with other work in the area (Van Os et al., 2002; Henquet et al., 2005), and is self-selected and thus prone to bias. Similarly, the sample is predominantly female, and this may affect the relationships observed as previous work suggests that women may be more likely to experience psychosis from frequent cannabis use (Compton et al., 2009). Second, the measure used here to assess cannabis use was not standardised, and a self-report measure of psychosis was used, whereas a diagnostic interview schedule has been used in previous research in the area (Henquet et al., 2005; Van Os et al., 2002). Third, due to the number of nonparametric t-tests here there is an increased risk of a type I error. However there was little alternative due to the skewed distribution of psychotic symptoms in this sample.

\section{Conclusion}

Despite these limitations the current findings are important as they suggest a relationship between cannabis use and increased psychotic symptoms in undergraduate students. As this is a population which has been identified as having very high levels of cannabis use, these results are important for university health clinics and clinicians who work with students as they suggest a need to screen for psychotic symptoms in students who have been identified as heavy users of cannabis. Similarly it is important to assess cannabis use in students presenting with psychotic 
illnesses. These results may also be of use for those developing interventions and prevention campaigns designed to reduce cannabis use in this population. Future research should examine this relationship using longitudinal designs and semistructured interviews. Doing so will help to give insight into the potential for psychotic symptoms to develop in a population who are high risk for cannabis use.

\section{Acknowledgements}

Thank you to the university staff who helped with recruitment and to those who took the time to complete the questionnaires. Thanks also to staff at the University of Bath whose comments have assisted in the preparation of this manuscript.

\section{References}

Arria, A.M., Caldeira, K.M., O'Grady, K.E., Vincent, K.B., Fitzelle, D.B., Johnson, E.P., \& Wish, E.D. (2008). Drug exposure opportunities and use patterns among college students: Results of a longitudinal prospective cohort study. Substance Abuse, 29(4), 19-38.

Arseneault, L., Cannon, M., Witton, J. \& Murray, R.M. (2004). Causal association between cannabis and psychosis: examination of the evidence. British Journal of Psychiatry, 184, 110-117.

Arseneault, L., Cannon, M., Poulton, R., Murray, R., Caspi, A. \& Moffitt, T.E. (2002). Cannabis use in adolescence and risk for adult psychosis: longitudinal prospective study. British Medical Journal, 325, 1212-1213.

Barkus, E. \& Lewis, S. (2008). Schizotypy and psychosis-like experiences from recreational cannabis in a non-clinical sample. Psychological Medicine, 38, 12671276.

Barnett, J.H., Werners, U., Secher, S.M., Hill, K.E., Brazil, R., Masson, K. et al. (2007). Substance use in a population-based clinic sample of people with firstepisode psychosis. British Journal of Psychiatry, 190, 515-520.

Beck, K.H., Caldeira, K.M., Vincent, K.B., O'Grady, K.E., Wish, E.D. \& Arria, A.M. (2009). The social context of cannabis use: Relationship to cannabis use disorders and depressive symptoms among college students. Addictive Behaviors, 34(9), 764-768.

Boydell, J., Van Os, J., Caspi, A., Kennedy, N., Giouroukou, E., Fearon, M. et al. (2006). Trends in cannabis use prior to first presentation with schizophrenia, in South-East London between 1965 and 1999. Psychological Medicine, 36, 14411446.

Caldeira, K.M., Arria, A.M., O'Grady, K.E., Vincent, K.B., \& Wish, E.D. (2008). The occurrence of cannabis use disorders and other cannabis-related problems among first-year college students. Addictive Behaviors, 33, 397-411.

Caspi, A., Moffitt, T.E., Cannon, M.E., McClay, J., Murray, R., Harrington, H.L. et al. (2005). Moderation of the Effect of Adolescent-Onset Cannabis Use on Adult Psychosis by a Functional Polymorphism in the Catechol-O-Methyltransferase Gene: Longitudinal Evidence of a Gene X Environment Interaction. Biological Psychiatry, 57, 117-1127. 
Compton, M.T. \& Ramsay, CE. (2009). The Impact of Pre-onset Cannabis Use on Age at Onset of Prodromal and Psychotic Symptoms. Primary Psychiatry, 16(4), 35-43.

Di Forti, M., Morgan, C., Dazzan, P., Pariante, C., Mondelli, V., Marques, T.R. et al. (2009). High-potency cannabis and the risk of psychosis. British Journal of Psychiatry, 195, 488-491.

D’Souza, D.C., Perry, E., MacDougall, L., Ammerman, Y., Cooper, T., Yu-Te, W. et al. (2004). The Psychotomimetic Effects of Intravenous Delta-9Tetrahydrocannabinol in Healthy Individuals: Implications for Psychosis. Neuropsychopharmacology, 29, 1558-1572.

Ferdinand, R.F., Sondeijker, F., van der Ende, J., Selten, J-P., Huizin, A. \& Verhulst, F.C. (2005). Cannabis use predicts future psychotic symptoms, and vice versa. Addiction, 100, 612-618.

Fergusson, D.M., Horwood, L.J. \& Swain-Campbell, N.R. (2003). Cannabis dependence and psychotic symptoms in young people. Psychological Medicine, $33,15-21$.

Hall, W. \& Degenhardt, L. (2009). Adverse health effects of non-medical cannabis use. The Lancet, 374, 1383-1391.

Hammersley, R. \& Leon, V. (2006). Patterns of cannabis use and positive and negative experiences of use amongst university students. Addiction Research and Theory, 14(2), 189-205.

Henquet, C., Krabbendam, L., Spauwen, J., Kaplan, C., Lieb, R., Wittchen, H-U. et al. (2005). Prospective cohort study of cannabis use, predisposition for psychosis, and psychotic symptoms in young people. British Medical Journal, 330, 11.

Hides, L., Lubman, D.I., Buckby, J., Yuen, H.P., Cosgrave, E. \& Baker, K. (2009). The association between early cannabis use and psychotic-like experiences in a community adolescent sample. Schizophrenia Research, 112, 130-135.

Hides, L., Dawe, S., Kavanagh, D.J. \& Young R.M. (2006). Psychotic symptom and cannabis relapse in recent-onset psychosis. British Journal of Psychiatry, 189, 137-143.

Hope, A., Dring, C. \& Dring, J. (2005). The health of Irish students: College Lifestyle and Attitudinal National (CLAN) Survey; a qualitative evaluation of the College Alcohol Policy Initiative. Health Promotion Unit, Department of Health and Children, Dublin.

Katz, G., Durst, R., Shufman, E., Bar-Hamburger, R. \& Grunhaus, L. (2010). Cannabis abuse and severity of psychotic and affective disorders in Israeli psychiatric inpatients. Comprehensive Psychiatry, 51(1), 37-41.

McLaren, J.A., Silins, E., Hutchinson, D., Mattick, R.P. \& Hall, W. (2010). Assessing evidence for a causal link between cannabis and psychosis: A review of cohort studies. International Journal of Drug Policy, 21, 10-19

Miettunen, J., Tormanen, S., Murray, G.K., Jones, P.B., Maki, P., Ebeling, H., Moilanen, H., Taanila, A., Heinimaa, M., Joukamaa M. \& Veijola, J. (2008). Association of cannabis use with prodromal symptoms of psychosis in adolescence. British Journal of Psychiatry, 192, 470-471.

National Advisory Committee on Drugs and Drugs and Alcohol Information and Research Unit (2003). Drug Use in Ireland and Northern Ireland: First results from 2002-2003.

Richardson, T. \& Gallagher, A. (2010). Letter to the editor: Comment on Hides et alCannabis use and psychotic-like experiences. Schizophrenia Research, 116, 98. 
Schofield, D., Tennant, C., Nash, L., Degenhardt, L., Cornish, A., Hobbs, C., \& Brenann, G. (2006). Reasons for cannabis use in psychosis. Australian and New Zealand Journal of Psychiatry, 40, 570-574.

Stefanis, N.C.P., Delespaul, H.C., Bakoula, C., Stefanis, C.N. \& Van O, J. (2005). Early adolescent cannabis exposure and positive and negative dimensions of psychosis. Addiction, 99, 1333-1341.

Stirling, J., Barkus, E.J., Nabosi, L., Irshad, S., Roemer, G., Schreudergoidheijt, B. \& Lewis, S. (2008). Cannabis-Induced Psychotic-Like Experiences Are Predicted by High Schizotypy: Confirmation of Preliminary Results in a Large Cohort. Psychopathology, 41, 371-378.

United Nations Office on Drugs and Crime. (2006). World drug report 2006. New York, United Nations.

Van Os, J., Linscott, R.J., Myin-Germeys, I., Delespaul, P. \& Krabbendam, L. (2009). A systematic review and meta-analysis of the psychosis continuum: evidence for a psychosis proneness-persistence-impairment model of psychotic disorder. Psychological Medicine, 39, 179-195.

Van Os, J., Bak, M., Hanssen, M., Bijl, R.V., de Graaf, R., \& Verdoux, H. (2002). Cannabis Use and Psychosis: A Longitudinal Population-based Study. American Journal of Epidemiology, 156(4), 319-327.

Verdoux, H., Gindre, C., Sorbara, F., Tourier, M. \& Swendsen, J.D. (2003). Effects of cannabis and psychosis vulnerability in daily life: an experience sampling test study. Psychological Medicine, 33, 23-32. 
Table I: Cannabis use variables in all participants and divided by gender

\begin{tabular}{|l|c|c|c|}
\hline & All participants & Male & Female \\
\hline Lifetime use & & & \\
Yes & $53.1 \%(\mathrm{n}=178)$ & $53.1 \%(\mathrm{n}=26)$ & $52.8 \%(\mathrm{n}=149)$ \\
No & $46.9 \%(\mathrm{n}=157)$ & $46.9 \%(\mathrm{n}=23)$ & $47.2 \%(\mathrm{n}=133)$ \\
\hline Current use & & & \\
Yes & $20.1 \%(\mathrm{n}=67)$ & $27.1 \%(\mathrm{n}=13)$ & $18.9 \%(\mathrm{n}=53)$ \\
No & $79.9 \%(\mathrm{n}=266)$ & $72.9 \%(\mathrm{n}=35)$ & $81.1 \%(\mathrm{n}=228)$ \\
\hline Frequency of use & & & \\
Yearly & $54.6 \%(\mathrm{n}=71)$ & $20 \%(\mathrm{n}=4)$ & $61.1 \%(\mathrm{n}=66)$ \\
Monthly & $25.4 \%(\mathrm{n}=33)$ & $45 \%(\mathrm{n}=9)$ & $21.3 \%(\mathrm{n}=23)$ \\
Weekly & $12.3 \%(\mathrm{n}=16)$ & $25 \%(\mathrm{n}=5)$ & $10.2 \%(\mathrm{n}=11)$ \\
Daily & $7.7 \%(\mathrm{n}=10)$ & $10 \%(\mathrm{n}=2)$ & $7.4 \%(\mathrm{n}=8)$ \\
\hline Addicted & & & \\
Yes & $2.2 \%(\mathrm{n}=4)$ & $0 \%(\mathrm{n}=0)$ & $2.7 \%(\mathrm{n}=4)$ \\
No & $96.1 \%(\mathrm{n}=171)$ & $96.2 \%(\mathrm{n}=25)$ & $96 \%(\mathrm{n}=143)$ \\
Not sure & $1.7 \%(\mathrm{n}=3)$ & $3.8 \%(\mathrm{n}=1)$ & $1.3 \%(\mathrm{n}=2)$ \\
\hline Age of first use & & & \\
Range & $10-38$ years old & $12-28$ years old & $10-38$ years old \\
Median & 16 years old & 16 years old & 16 years old \\
\hline 'Joints' in past year & & & \\
Range & 0 to 600 'joints' & 0 to 600 'joints' & 0 to 500 'joints' \\
Median & 2 'joints' & 2 'joints'* & 2 'joints' \\
\hline 'Joints' in lifetime & & & \\
Range & $0-30,000$ 'joints' & $0-2,000$ 'joints' & $0-30,000$ 'joints' \\
Median & 14 'joints' & 50 'joints'** & 10 'joints' \\
\hline
\end{tabular}

$*=$ Significantly higher for males; $\mathrm{p}<.05$

$* *=$ Significantly higher for males; $\mathrm{p}<.01$ 
Figure 1: Distribution of total scores on the DIS-P

**See attached Figure $* *$ 


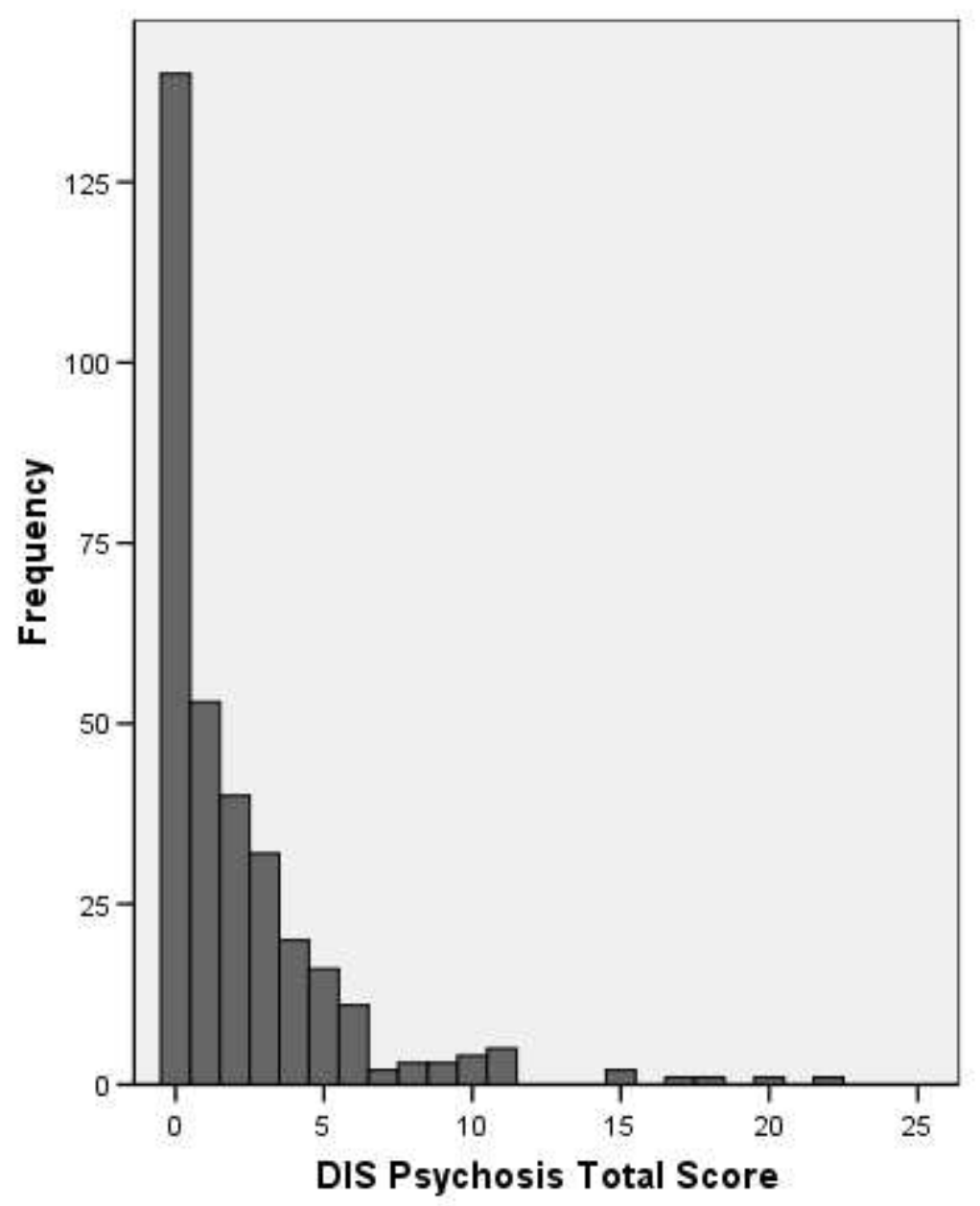

$115 \times 132 \mathrm{~mm}(96 \times 96 \mathrm{DPI})$

2

3

4

5

6
7

8

9

10

11

12

13

14
15

16

17

18

19

20

21

23

24

25

26

27

28

30

31

32

33

34

35

36

40

41

42

43

44

45

46

47

48

49

50

51

52

53

54

55

56

57

58

59

60

URL: http:/mc.manuscriptcentral.com/rpsy 